PROCEEDINGS OF THE

AMERICAN MATHEMATICAL SOCIETY

Volume 127, Number 11, Pages 3421-3426

S 0002-9939(99)04924-2

Article electronically published on May 27, 1999

\title{
VANISHING OF A CERTAIN KIND OF VASSILIEV INVARIANTS OF 2-KNOTS
}

\author{
SEIICHI KAMADA
}

(Communicated by Ronald A. Fintushel)

\begin{abstract}
In knot theory, Vassiliev's 1-knot invariants are defined in a combinatorial way as finite type invariants. By a natural generalization of the combinatorial definition, one has a certain family of 2-knot invariants, which should be called finite type 2-knot invariants. They form a subspace of the whole space of "Vassiliev 2-knot invariants". In this paper we prove that it is 1-dimensional.
\end{abstract}

By a $\Sigma$-immersion, we mean an immersion $k: \Sigma \rightarrow R^{4}$ of an oriented, closed and connected 2-manifold $\Sigma$ into 4 -space. If there exist orientation-preserving diffeomorphisms of $\Sigma$ and $R^{4}$ to themselves which make the following diagram commutative, then we say that $k$ and $k^{\prime}$ are equivalent:

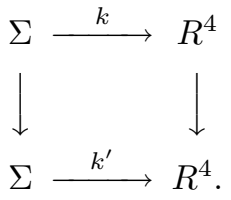

A $\Sigma$-immersion is generic if the singularities are transverse double points. In this case, the normal Euler number $e(k)$ of $k$ is equal to $2\left(d_{+}(k)-d_{-}(k)\right)$, where $d_{+}(k)$ is the number of positive double points of $k$ and $d_{-}(k)$ is that of negative ones.

By the Smale and Hirsch theorem [10], [11], [4], two $\Sigma$-immersions $k_{0}$ and $k_{1}$ are related by a regular homotopy if and only if $e\left(k_{0}\right)=e\left(k_{1}\right)$. Moreover, if $k_{0}$ and $k_{1}$ are generic, then by taking a regular homotopy generically, we have a generic one-parameter family of $\Sigma$-immersions $k_{t}(t \in[0,1])$ being generic for all but a finite number of values $t$ such that at each exceptional $t$ a finger move or a Whitney trick occurs. (There exists an explicit procedure to construct such a one-parameter family, cf. [6].) A finger move (cf. [3], [7], [8], [9]) is a regular homotopy between $\Sigma$-immersions yielding a pair of positive and negative transverse double points, whose local image is illustrated in Figure 1 in the motion picture method. The inverse operation is a Whitney trick in dimension four. It is a difficult problem to determine whether or not a given pair of double points is a Whitney pair (cf. [3], [7], [9]).

Let $\mathcal{K}(\Sigma)_{(n)}$ be the set of equivalence classes of $\Sigma$-immersions whose singularities are (transverse or non-transverse) double points and the number of non-transverse

Received by the editors May 22, 1997 and, in revised form, February 1, 1998.

1991 Mathematics Subject Classification. Primary 57Q45, 57M25.

Key words and phrases. 2-knot, Vassiliev invariant, finite type invariant, finger move, regular homotopy. 


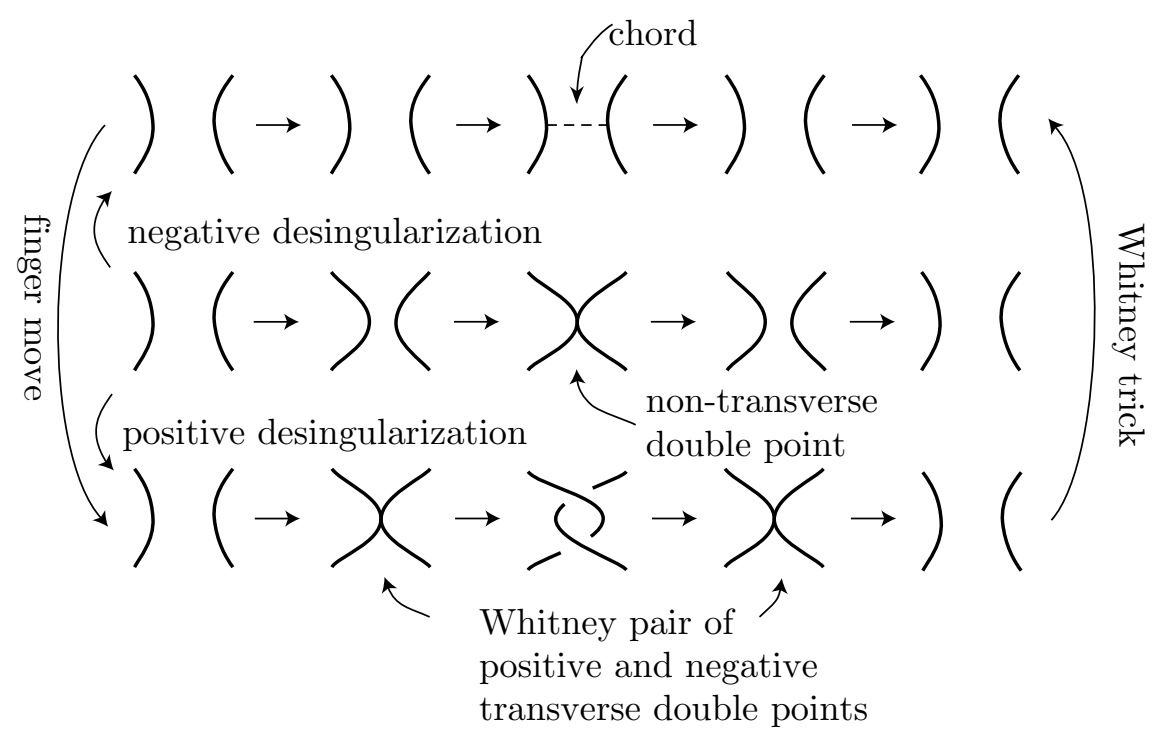

Figure 1

ones is $n$. In particular, $\mathcal{K}(\Sigma)_{(0)}$ (denoted simply by $\mathcal{K}(\Sigma)$ ) is the set of equivalence classes of generic $\Sigma$-immersions. If a $\Sigma$-immersion $k$ has a non-transverse double point, then there exists a finger move (regular homotopy) $k_{t}(t \in[0,1])$ with $k_{0.5}=k$ as a bifurcation. We call the restricted homotopy $k_{t}(t \in[0.5,1])$ a positive desingularization, and the inverse of the restricted homotopy $k_{t}(t \in[0,0.5])$ a negative desingularization. The equivalence classes of $k_{0}$ and $k_{1}$ are uniquely determined from that of $k$.

Let $v: \mathcal{K}(\Sigma) \rightarrow \mathrm{F}$ be a mapping of $\mathcal{K}(\Sigma)$ to a field $\mathrm{F}$ (which may be a commutative ring with unit or an abelian group). For a $\Sigma$-immersion $k_{\times}$whose class $\left[k_{\times}\right]$belongs to $\mathcal{K}(\Sigma)_{(1)}$, define $v\left(\left[k_{\times}\right]\right)$by $v\left(\left[k_{+}\right]\right)-v\left(\left[k_{-}\right]\right)$, where $k_{+}$and $k_{-}$are $\Sigma$-immersions obtained from $k_{\times}$by positive and negative desingularizations respectively. Inductively, the mapping $v: \mathcal{K}(\Sigma) \rightarrow \mathrm{F}$ is extended to $v: \amalg_{n=0}^{\infty} \mathcal{K}(\Sigma)_{(n)} \rightarrow \mathrm{F}$. (It seems reasonable that such a mapping is called Vassiliev's invariant of $\Sigma$ immersions, cf. [1]. The family of such mappings forms a linear space over F.) If there exists an integer $n_{0}$ such that $v$ vanishes over all $\mathcal{K}(\Sigma)_{(n)}$ with $n \geq n_{0}$, we say that $v$ is of finite type.

Theorem 1. Let $v: \mathcal{K}(\Sigma) \rightarrow \mathrm{F}$ be a mapping of finite type. Then $v([k])=v\left(\left[k^{\prime}\right]\right)$ for any generic $\Sigma$-immersions $k$ and $k^{\prime}$ with $e(k)=e\left(k^{\prime}\right)$ and $d(k)=d\left(k^{\prime}\right)$.

Here $d(k)$ stands for the number of (transverse) double points of $k$. The assumption of the theorem is equal to $d_{+}(k)=d_{+}\left(k^{\prime}\right)$ and $d_{-}(k)=d_{-}\left(k^{\prime}\right)$.

Consider a special case that $\Sigma$ is a 2 -sphere $S^{2}$. We call a 2 -knot invariant valued over $\mathrm{F}$ a finite type invariant if it is extended to a mapping $v: \mathcal{K}\left(S^{2}\right) \rightarrow \mathrm{F}$ of finite type. This is a natural analogue of finite type 1-knot invariants and Vassiliev's invariants in knot theory. Theorem 1 implies the following theorem.

Theorem 2. The set of finite type 2-knot invariants is 1-dimensional, i.e., they are constant maps. 


\section{The Vassiliev module of $\Sigma$-IMMERsions}

Let $k$ be a $\Sigma$-immersion whose class $[k]$ belongs to $\mathcal{K}(\Sigma)_{(n)}$ and $v_{1}, \ldots, v_{n}$ the non-transverse double points of $k$. For each $n$-tuple of signs $\left(\epsilon_{1}, \ldots, \epsilon_{n}\right)$, let $k_{\epsilon_{1}, \ldots, \epsilon_{n}}$ denote a generic $\Sigma$-immersion obtained by desingularizations on $v_{1}, \ldots, v_{n}$ in directions according to $\left(\epsilon_{1}, \ldots, \epsilon_{n}\right)$. Then an element

$$
\sum_{\left(\epsilon_{1}, \ldots, \epsilon_{n}\right)} \epsilon_{1} \ldots \epsilon_{n}\left[k_{\epsilon_{1}, \ldots, \epsilon_{n}}\right] \in \mathbb{Z K} \mathcal{K}(\Sigma)
$$

is determined from $[k]$ up to sign. Denote by $L_{n}$ the subspace of $\mathbb{Z} \mathcal{K}(\Sigma)$ spanned by such elements for all $[k] \in \mathcal{K}(\Sigma)_{(n)}$. Evidently, we have $L_{1} \supset L_{2} \ldots$ The Smale and Hirsch theorem implies that $[k]-\left[k^{\prime}\right] \in L_{1}$ if and only if $e(k)=e\left(k^{\prime}\right)$. The $n$-th Vassiliev module is the $\left(\mathbb{Z}\right.$-) module $\mathbb{Z} \mathcal{K}(\Sigma) / L_{n}$. (By tensor product with $\mathrm{F}$, one may consider it a vector space or a module over $\mathrm{F}$.) A mapping $v: \mathcal{K}(\Sigma) \rightarrow \mathrm{F}$ is of finite type if and only if its extension $v: \mathbb{Z K}(\Sigma) \rightarrow \mathrm{F}$ is factored through this module for some $n$.

The following is our main theorem, which implies Theorem 1.

Theorem 3. Let $k$ and $k^{\prime}$ be generic $\Sigma$-immersions with $e(k)=e\left(k^{\prime}\right)$ and $d(k)=$ $d\left(k^{\prime}\right)$. Then $[k]-\left[k^{\prime}\right] \in L_{n}$ for every $n \in \mathbb{N}$.

\section{UNKNOTTED $\Sigma$-IMMERSIONS}

A generic $\Sigma$-immersion is called an unknotted one if it is equivalent to a standard embedding of $\Sigma$ with some (or no) "trivial kinks" (see Figure 2). We denote by $u_{d}^{e}$ an unknotted $\Sigma$-immersion with $e\left(u_{d}^{e}\right)=e$ and $d\left(u_{d}^{e}\right)=d$. Notice that the equivalence class of $u_{d}^{e}$ is unique.

Let $k: \Sigma \rightarrow R^{4}$ be a $\Sigma$-immersion. A chord $\gamma$ attached to $k(\Sigma)$ means a smooth simple arc in $R^{4}$ whose endpoints are distinct points of $k(\Sigma)$ except the singularities and the interior of $\gamma$ is disjoint from it. By a standard argument on general position, the ambient isotopy class of $\gamma$ by isotopies of $R^{4}$ keeping $k(\Sigma)$ setwise fixed may be treated as a homotopy class in the sense of [2], [5]. If $k^{\prime}$ is a $\Sigma$-immersion obtained from $k$ by a finger move along $\gamma$ (see Figure 1), then $\left[k^{\prime}\right]$ is uniquely determined from $[k]$ and the class of $\gamma$. (The $\Sigma$-immersion appearing as a bifurcation in the finger move is obtained from $k$ by shrinking $\gamma$.)

Lemma 1. Let $u$ be an unknotted $\Sigma$-immersion. Any $\Sigma$-immersion obtained from $u$ by a finger move is also unknotted.

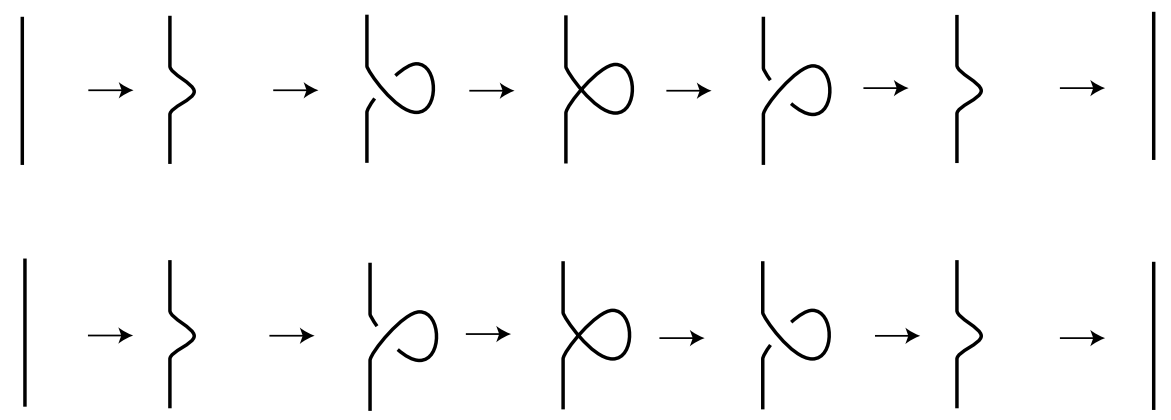

Figure 2 


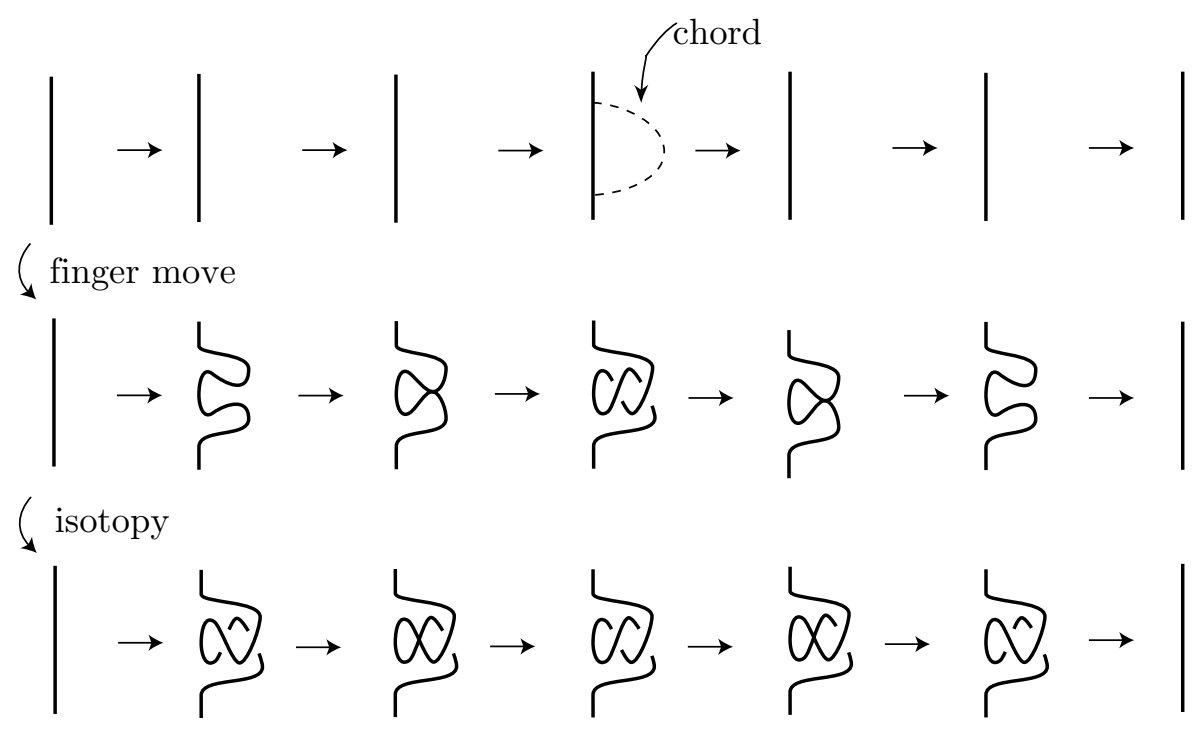

FIGURE 3

Proof. Let $\gamma$ be a chord attached to $u(\Sigma)$ along which the finger move is applied. Since the fundamental group $\pi_{1}\left(R^{4} \backslash u(\Sigma), *\right)$ is an infinite cyclic group generated by the meridian of $u(\Sigma)$, by arguments of [5], [2], we may assume that $\gamma$ is a chord attached to $u(\Sigma)$ trivially. The finger move brings the $\Sigma$-immersion $u$ a pair of positive and negative trivial kinks, see Figure 3.

Lemma 2. If a $\Sigma$-immersion $k^{\prime}$ is obtained from another $k$ by a Whitney trick followed by a finger move, then it is also obtained, up to equivalence, from $k$ by a finger move followed by a Whitney trick.

Proof. Let $V$ be a 4-ball in $R^{4}$ such that a Whitney trick performed in $V$ as in Figure 1 changes $k$ into a $\Sigma$-immersion $k^{\prime \prime}$ and a finger move along a chord $\gamma$ attached to $k^{\prime \prime}(\Sigma)$ changes $k^{\prime \prime}$ into $k^{\prime}$. If $\gamma$ is disjoint from $V$, the assertion is obvious. Since the inclusion-induced homomorphism $\pi_{1}\left(\partial V \backslash k^{\prime \prime}(\Sigma), *\right) \rightarrow \pi_{1}\left(V \backslash k^{\prime \prime}(\Sigma), *\right)$ is surjective (in fact, isomorphic), we may assume that $\gamma$ is disjoint from $V$.

Lemma 3 (Unknotting Lemma). For any generic $\Sigma$-immersion $k$, there exists a family of mutually disjoint chords attached to $k(\Sigma)$ such that finger moves along them change $k$ into an unknotted one

Proof. By the Smale and Hirsch theorem, there exists a generic regular homotopy between $k$ and an unknotted $\Sigma$-immersion $u$. Using the previous lemma, we see that there exists a $\Sigma$-immersion $k^{\prime}$ such that (i) it is obtained from $k$ by a sequence of finger moves and (ii) it is obtained also from $u$ by a sequence of finger moves. By (ii) and Lemma $1, k^{\prime}$ is unknotted. By a similar argument as in the proof of Lemma 2, it is easily seen that a sequence of finger moves may be replaced with the same number of simultaneous finger moves. Thus, from (i), we have the result.

The unknotting number of a generic $\Sigma$-immersion $k$ is defined by the minimum number of chords as in Lemma 3, which we denote by $\mathrm{u}(k)$. By the proof of 
Lemma 3, we see that the unknotting number $\mathrm{u}(k)$ equals the minimum number of finger moves appearing in generic regular homotopies between $k$ and unknotted $\Sigma$-immersions.

\section{Proof of Theorem 3}

Let $k$ be a generic $\Sigma$-immersion and $\gamma_{1}, \ldots, \gamma_{n}$ mutually disjoint chords attached to $k(\Sigma)$. For each $n$-tuple of signs $\left(\epsilon_{1}, \ldots, \epsilon_{n}\right)$, let $k_{\epsilon_{1}, \ldots, \epsilon_{n}}$ denote a generic $\Sigma$ immersion obtained by finger moves along $\gamma_{i}$ for $i$ with $\epsilon_{i}=+1$ and by eliminating $\gamma_{i}$ for $i$ with $\epsilon_{i}=-1$. When one considers a $\Sigma$-immersion with $n$ non-transverse double points obtained from $k$ by shrinking the chords, $k_{\epsilon_{1}, \ldots, \epsilon_{n}}$ is the same as before. Thus, in this situation, we have

$$
\sum_{\left(\epsilon_{1}, \ldots, \epsilon_{n}\right)} \epsilon_{1} \ldots \epsilon_{n}\left[k_{\epsilon_{1}, \ldots, \epsilon_{n}}\right] \in L_{n}
$$

Proof of Theorem 3. It is sufficient to prove the following assertion for every $m \in \mathbb{N}$.

Assertion $(\mathrm{m})$. Let $k$ and $k^{\prime}$ be generic $\Sigma$-immersions with $e(k)=e\left(k^{\prime}\right)$ and $d(k)=d\left(k^{\prime}\right)$. If $\mathrm{u}(k) \leq m$ and $\mathrm{u}\left(k^{\prime}\right) \leq m$, then $[k]-\left[k^{\prime}\right] \in L_{n}$ for any $n \geq m+1$.

Since $\mathrm{u}(k) \leq m$, there exist $m$ mutually disjoint chords attached to $k(\Sigma)$, say $\gamma_{1}, \ldots, \gamma_{m}$, such that the finger moves along them change $k$ into an unknotted one, which is $u_{d+2 m}^{e}$ where $e=e(k)$ and $d=d(k)$. Let $n$ be an integer with $n \geq m+1$, and take parallel copies $\gamma_{m+1}, \ldots, \gamma_{n}$ of $\gamma_{1}$ such that $\gamma_{1}, \ldots, \gamma_{n}$ are mutually disjoint chords attached to $k(\Sigma)$. For each $n$-tuple of signs $\left(\epsilon_{1}, \ldots, \epsilon_{n}\right)$, let $k_{\epsilon_{1}, \ldots, \epsilon_{n}}$ denote a generic $\Sigma$-immersion obtained by finger moves along $\gamma_{i}$ for $i$ with $\epsilon_{i}=+1$ as before. Let $p=p\left(\epsilon_{1}, \ldots, \epsilon_{n}\right)$ be the number of positive signs. If $p$ is not zero, then by Lemma 1 we have $\mathrm{u}\left(k_{\epsilon_{1}, \ldots, \epsilon_{n}}\right) \leq m-1, e\left(k_{\epsilon_{1}, \ldots, \epsilon_{n}}\right)=e$ and $d\left(k_{\epsilon_{1}, \ldots, \epsilon_{n}}\right)=d+2 p$. We use the induction on $m$. If $m=1$, then $k_{\epsilon_{1}, \ldots, \epsilon_{n}}=u_{d+2 p}^{e}$ for any $\left(\epsilon_{1}, \ldots, \epsilon_{n}\right)$ with $p=p\left(\epsilon_{1}, \ldots, \epsilon_{n}\right) \neq 0$. Thus,

$$
\sum_{\left(\epsilon_{1}, \ldots, \epsilon_{n}\right)} \epsilon_{1} \ldots \epsilon_{n}\left[k_{\epsilon_{1}, \ldots, \epsilon_{n}}\right]=\sum_{p=1}^{n}(-1)^{n-p}\left(\begin{array}{l}
n \\
p
\end{array}\right)\left[u_{d+2 p}^{e}\right]+(-1)^{n}[k] .
$$

Since the left-hand side belongs to $L_{n}$ and a similar equation holds for $k^{\prime}$, we have $[k]-\left[k^{\prime}\right] \in L_{n}$. If $m \geq 2$, then by the induction hypothesis we have $\left[k_{\epsilon_{1}, \ldots, \epsilon_{n}}\right]-$ $\left[u_{d+2 p}^{e}\right] \in L_{n}$ for any $\left(\epsilon_{1}, \ldots, \epsilon_{n}\right)$ with $p=p\left(\epsilon_{1}, \ldots, \epsilon_{n}\right) \neq 0$. Thus,

$$
\sum_{\left(\epsilon_{1}, \ldots, \epsilon_{n}\right)} \epsilon_{1} \ldots \epsilon_{n}\left[k_{\epsilon_{1}, \ldots, \epsilon_{n}}\right] \equiv \sum_{p=1}^{n}(-1)^{n-p}\left(\begin{array}{l}
n \\
p
\end{array}\right)\left[u_{d+2 p}^{e}\right]+(-1)^{n}[k] \quad\left(\bmod L_{n}\right) .
$$

Therefore, we see that $[k]-\left[k^{\prime}\right] \in L_{n}$.

The author would like to thank the referee for informing him that Tobias Ekholm has considered a similar problem under the direction of Oleg Viro.

\section{REFERENCES}

[1] J. S. Birman and X.-S. Lin, Knot polynomials and Vassiliev's invariant, Invent. Math. 11 (1993), 225-270. MR 94d:57010

[2] J. Boyle, Classifying 1-handles attached to knotted surfaces, Trans. Amer. Math. Soc. 306 (1988), 475-487. MR 89f:57032

[3] A. J. Casson, Three lectures on new-infinite constructions in 4-dimensional manifolds, A la Recherche de la Topologie Perdue, Progress in Math., 62, Birkhäuser, 1986, pp. 201-214. CMP 19:16 
[4] M. W. Hirsch, Immersions of manifolds, Trans. Amer. Math. Soc. 93 (1959), 242-276. MR 22:9980

[5] F. Hosokawa and A. Kawauchi, Proposals for unknotted surfaces in four-spaces, Osaka J. Math. 16 (1979), 233-248. MR 81c:57018

[6] S. Kamada, Unknotting operations for 1- and 2-dimensional links, The Proceedings of the fifth Korea-Japan School of Knots and Links, held in Taejon, Korea, 1997, to appear.

[7] R. Kirby, The topology of 4-manifolds, Lecture Notes in Mathematics, 1374, Springer-Verlag, 1989. MR 90j:57012

[8] P. Kirk, Link maps in the four sphere, Differential Topology, Lect. Notes Math., 1350, Springer-Verlag, 1988, pp. 31-43. MR 90e:57043

[9] G.-S. Li, An invariant of link homotopy in dimension four, Topology 36 (1997), 881-897. MR 98g:57038

[10] S. Smale, A classification of immersions of the two-sphere, Trans. Amer. Math. Soc. 90 (1959), 281-290. MR 21:2984

[11] — The classification of immersions of spheres in Euclidean spaces, Ann. Math. 69 (1959), 327-344.

[12] V. A. Vassiliev, Cohomology of knot space, Theory of Singularities and Its Applications, Advances in Soviet Math. vol. 1, Amer. Math. Soc., 1990. MR 92a:57016

Department of Mathematics, Osaka City University, Sumiyoshi-ku, Osaka 558, Japan

E-mail address: kamada@sci.osaka-cu.ac.jp 